\section{Kidney \\ Blood Pressure Research}

\title{
Early Start Peritoneal Dialysis: Technique Survival in Long-Term Follow-Up
}

\author{
Bruno C. Silva ${ }^{\mathrm{a}}$ Erica Adelina $^{\mathrm{a}}$ Benedito J. Pereira $^{\mathrm{a}}$ Lilian Cordeiro $^{\mathrm{a}}$ \\ Camila E. Rodrigues $^{a}$ Ricardo J. Duarte ${ }^{b}$ Hugo Abensur ${ }^{a}$ Rosilene M. Elias ${ }^{a, c}$ \\ aDepartment of Medicine, Renal Division, Hospital das Clinicas HCFMUSP, Universidade de São Paulo, \\ São Paulo, bUrology Division, Hospital das Clinicas HCFMUSP, Universidade de São Paulo, São Paulo, \\ Universidade Nove de Julho (UNINOVE), São Paulo, Brazil
}

\section{Key Words}

Peritoneal dialysis • Unplanned peritoneal dialysis • Acute peritoneal dialysis

\begin{abstract}
Background/Aims: Peritoneal dialysis (PD) has gained interest over the last decade as a viable option for early start dialysis. It is still unknown if shorter break-in periods and less time for proper patient evaluation and training could influence technique survival in comparison to planned-start PD. Methods: A prospective and observational study that compared technique survival in a cohort of patients who started either early or planned PD. Early start PD was defined as break-in period from 3 to 14 days with no previous nephrologist follow-up or patient training. Results: A total of 154 patients were included (40 as early start PD), followed by a median time of 381 days. Comparing early vs. planned-start PD, groups were similar concerning age $56(40 ; 70)$ vs. $48(32 ; 63)$ years, $p=0.071$, body mass index (BMI) $23.3 \pm 4.2$ vs. $23.8 \pm 4.0 \mathrm{~kg} / \mathrm{m}^{2}, \mathrm{p}=0.567$ and male gender (60 vs. 48\%, $\mathrm{p}=0.201$ ), respectively. Comparing early vs. planned-start groups, there were no differences regarding PD dropout for peritonitis (7.5 vs. $11.4 \%, p=0.764)$, catheter dysfunction (12.5 vs. $17.5 \%, p=0.619$ ) and patient burnout (0 vs. $4.4 \%, p=0.328$ ), respectively. Less patients in early start group quit PD for peritoneal membrane failure in comparison to planned-start group ( $2.5 \mathrm{vs.} 16.7 \%, p=0.026)$. In multivariate cox-regression analysis, the only factors independently associated with technique failure were $B M I>25 \mathrm{~kg} / \mathrm{m}^{2}(p=0.033)$ and Diabetes Mellitus $(p=0.013)$, whereas no differences regarding early vs. planned-PD start were observed $(p=0.184)$. Conclusion: Despite the adverse scenario for initiating dialysis, early start PD had similar outcomes in comparison to planned-start PD in long-term follow-up.




\section{Kidney Blood Pressure Research}

Silva et al.: Early Start PD Technique Survival

\section{Introduction}

Peritoneal dialysis (PD) is a well-established treatment for end-stage renal disease (ESRD). Even though such statement is widely accepted, it is only valid in a planned background, when patient is adequately followed-up by a multi-professional team, with proper training and, most importantly, with a reliable and timely insertion of peritoneal catheter [1].

The difficulties of combining all these factors might partially explain why hemodialysis (HD) through a central venous catheter (CVC) is considered the default renal replacement therapy in the context of urgent-start dialysis. Other contributing factors are the relative difficulty of obtaining a rapid insertion of a peritoneal catheter, concerns regarding possible complications associated with short break-in periods for starting PD and the relative ease of CVC insertions [2]. However, starting unplanned HD using CVC is independently associated with bloodstream infection and mortality in incident dialysis patients [3, 4]. Yet, the risk of septicemia is similar for PD catheters and arteriovenous fistula [3].

In the last decade, many studies have provided enough evidence to support PD as a feasible therapy for urgent/early start dialysis. They have reported an overall low risk for mechanical complications or peritonitis [5-11]. However, most studies that compared planned vs. urgent/early start PD have focused on short-term outcome measures.

Starting PD at the emergency environment is a stressful event, far from ideal conditions. Shorter training and break-in periods and possibly inaccurate clinical and social evaluation could lead to more technique failure or burnout at long-term. For this purpose, we conducted a study that aimed to evaluate technique survival in a cohort of patients that have started either early or planned-PD.

\section{Materials and Methods}

\section{Statement of ethics}

All patients gave written informed consent. This study was approved by local ethics committee (CAPpesq \#45163715400000068).

\section{Patients}

Patients were divided into 2 groups: early start PD and planned-start PD. They were enrolled in this prospective and observational study from December 2010 to January 2018 at Hospital das Clínicas, University of São Paulo. Early start PD was defined as break-in period from 3 to 14 days after catheter insertion, with no previous nephrologist follow-up or patient/family training. Planned-start PD was defined as break-in period > 14 days after catheter placement, regardless of previous nephrologist follow-up.

The PD catheter used was a straight, double-cuffed Tenckhoff catheter, inserted either percutaneously by nephrologists (Seldinger technique) or videolaparoscopically by surgeons.

All patients were treated with lactate-buffered glucose dialysate. PD was performed either by Homechoice $^{\mathrm{TM}}$ automated cycler machine (Baxter Inc., Deerfield, USA) or PD-NIGHT Peritoneal Dialysis Machine $^{\mathrm{TM}}$ (Fresenius Medical Care, Brazil). In early start group, dwell volume was $1000 \mathrm{~mL}$ during the first week and was gradually increased up to $2000 \mathrm{~mL}$ over the first month, whereas in planned-start PD dwell volume ranged from 1500 to $2000 \mathrm{ml}$.

\section{Outcomes}

The main outcome was technique survival comparing early start and planned-start PD patients. Technique failure was defined as transitioning from PD to HD due to the following causes: peritoneal membrane failure, peritonitis, catheter dysfunction and patient burnout. 


\section{Kidney Blood Pressure Research}

Follow-up

All patients were followed over the study period. The following outcomes were assessed in the remaining patients: death, kidney transplantation, renal function recovery and technique failure.

\section{Statistical analysis}

Normally distributed variables are expressed as mean \pm standard deviation, while skewed variables are expressed and median and $(25 ; 75)$ percentiles. Categorical variables are expressed as percentage. Comparisons between groups were evaluated by unpaired $t$ test, Mann-Whitney test or Chi-square test, as appropriate. Technique survival was evaluated in whole group and also stratified by early or planned-start PD by Kaplan-Meier and Cox regression analyses. Statistical analysis was performed using SPSS software package, v. 22 (SPSS Inc., Chicago, IL, USA).

\section{Results}

\section{Patients characteristics and follow-up}

A total of 154 patients initiated PD during study period (40 as early start PD). Baseline characteristics of the population are described in Table 1. Median follow-up time was 381 days. Patients have been included in early start PD more frequently since 2014 in our service, and therefore the follow-up period was slightly lower in this group in comparison to planned-start group. The etiology of ESRD was different between groups, as heart failure was significantly more prevalent in early start group $(p=0.016)$. Nine patients in this group had no defined etiology of ESRD, whereas only 10 in planned-start PD group $(\mathrm{p}=0.023)$. Nephrologists inserted more catheters in planned-start in comparison to early start group. Four patients from early start group and eight patients from planned-start group had early technique failure, defined as PD dropout within 3 months after dialysis initiation. There were no differences regarding dialysis indication between groups.

\section{Urgent vs. planned PD}

As shown in Table 2, there were no differences in kidney transplantation and renal function recovery rates when comparing early start and plannedstart groups. Technique failure was significantly more observed in planned PD group, as a result of more cases of peritoneal membrane failure $(2.8 \%$

Table 1. Characteristics of study population, according to PD initiation. BMI: body mass index, GN: glomerulonephritis, DM: Diabetes Mellitus, AH: arterial hypertension, ADPKD: autossomic dominant polycystic kidney disease. * $\mathrm{p}<0.05$ between groups, CKD-MBD: chronic kidney disease-mineral and bone disorder

\begin{tabular}{lcccc}
\hline Variable & Whole cohort & Early start PD (n=40) & Planned-start PD (n=114) & $\mathrm{p}$ \\
\hline Age (years) & $50(34 ; 65)$ & $56(40 ; 70)$ & $48(32 ; 63)$ & 0.071 \\
Follow-up (days) & $381(179 ; 757)$ & $219(115 ; 642)$ & $418(197 ; 810)$ & 0.053 \\
BMI $\left(\mathrm{kg} / \mathrm{m}^{2}\right)$ & $23.7 \pm 4.1$ & $23.3 \pm 4.2$ & $23.8 \pm 4.0$ & 0.567 \\
Gender $\%$ male) & 51 & 60 & 48 & 0.201 \\
Catheter inserted by & 70 & 56 & 77 & 0.012 \\
nephrologists (\%) & & & & 0.025 \\
Etiology (\%) & 28.6 & 27.5 & 28.9 & \\
$\quad$ GN & 25.3 & 20 & 27.3 & \\
DM & 23.4 & 17.5 & 25.4 & \\
AH & 5.8 & 5 & 6.1 & \\
Urological & 2.6 & 0 & 3.5 & \\
$\quad$ Nephrotoxicity & 1.3 & 5 & 0 & \\
Heart Failure* & 12.3 & 22.5 & 8.8 & \\
$\quad$ Unknown* & 0.6 & 2.5 & 0 & \\
$\quad$ ADPKD & & & & \\
Dialysis indication (\%) & 71 & 73 & 70 & \\
$\quad$ Uremia & 24 & 27 & 7 & \\
$\quad$ Fluid overload & 5 & 0 & & \\
$\quad$ Complications of CKD-MBD & 5 & & & \\
\end{tabular}

Table 2. Outcomes, according to PD initiation. pts: patients, HD: hemodialysis, PM: peritoneal membrane

\begin{tabular}{lcccc}
\hline Outcomes & Whole cohort & Early start PD (n=40) & Planned-start PD (n=114) & $\mathrm{p}$ \\
\hline Deaths (\%) & 7.1 & 10 & 6.1 & 0.477 \\
Kidney transplantation (\%) & 14.3 & 5.0 & 17.5 & 0.065 \\
Renal function recovery (\%) & 3.9 & 5.0 & 3.5 & 0.650 \\
Conversion to HD (\%) & 36.4 & 15 & 43.9 & 0.001 \\
Time for HD conversion (days) & 365 & 300 & 365 & 0.652 \\
& $(214 ; 821)$ & $(183 ; 808)$ & $(208 ; 836)$ & \\
Peritonitis (\%) & 10.4 & 7.5 & 11.4 & 0.764 \\
Time for peritonitis (days) & 493 & 749 & 478 & 1.000 \\
PM. failure (\%) & $(262 ; 881)$ & $(214 ; 987)$ & $(271 ; 873)$ & 0.026 \\
Catheter dysfunction (\%) & 13 & 2.5 & 16.7 & 0.619 \\
Burnout (\%) & 16.2 & 12.5 & 17.5 & 0.328 \\
Catheter replacement (\%) & 3.2 & 0 & 4.4 & 0.042 \\
\hline
\end{tabular}




\section{Kidney Blood Pressure Research}

vs $17.9 \%$ in early start and planned-start PD groups, respectively, $\mathrm{p}=0.024)$. PD dropout for peritonitis, catheter dysfunction and burnout were similar between groups. Catheter replacement was higher in planned-start PD group. Deaths were similar between groups: 11.1\% in early start and $6.6 \%$ in planned-start PD ( $\mathrm{p}=0.382)$.

\section{Technique failure}

At univariate analysis (Table 3), patients who presented technique failure were more likely to have Diabetes Mellitus (DM). Technique failure was more frequent in the plannedstart PD group.

The analysis of technique failure according to PD start group, censored by death, kidney transplantation and renal function recovery obtained by Kaplan-Meier curve showed no differences between groups (Log-Rank test, $\chi^{2}=2.187, \mathrm{p}=0.139$ ), as shown in Fig. 1a. In Cox regression analysis, only overweight, defined as body mass index (BMI) > $25 \mathrm{~kg} / \mathrm{m}^{2}$, and $\mathrm{DM}$ were statistically associated with technique failure (Table 4 and Fig. 1b). Early technique failure, defined as catheter dysfunction less than 90 days after catheter placement was observed in 4 patients from early-start group and in 8 patients from planned-start group $(\mathrm{p}=0.51)$. Additionally, there was no difference regarding

catheter placement techniques (surgical or percutaneous) in these 12 patients ( 6 for each group).
Kidney Blood Press Res 2018;43:1699-1705

\begin{tabular}{l|l}
\hline DOI: 10.1159/000495386 & $\odot 2018$ The Author(s). Published by S. Karger AG, Basel
\end{tabular}

Published online: 23 November 2018 www.karger.com/kbr
Table 3. Univariate analysis: risk factors for technique failure. BMI: body mass index

\begin{tabular}{lccc}
\hline Covariate & $\begin{array}{c}\text { Technique failure } \\
(\mathrm{n}=56)\end{array}$ & $\begin{array}{c}\text { No technique failure } \\
(\mathrm{n}=98)\end{array}$ & $\mathrm{p}$ \\
\hline Age (years) & $53(31 ; 65)$ & $49.5(35 ; 65)$ & 0.377 \\
BMI $>25 \mathrm{~kg} / \mathrm{m}^{2}(\%)$ & 46.3 & 31.9 & 0.111 \\
Diabetes Mellitus (\%) & 35.7 & 19.4 & 0.034 \\
Early start PD (\%) & 10.7 & 34.7 & 0.001 \\
Catheter inserted by nephrologists (\%) & 76.8 & 66.3 & 0.118 \\
\hline
\end{tabular}

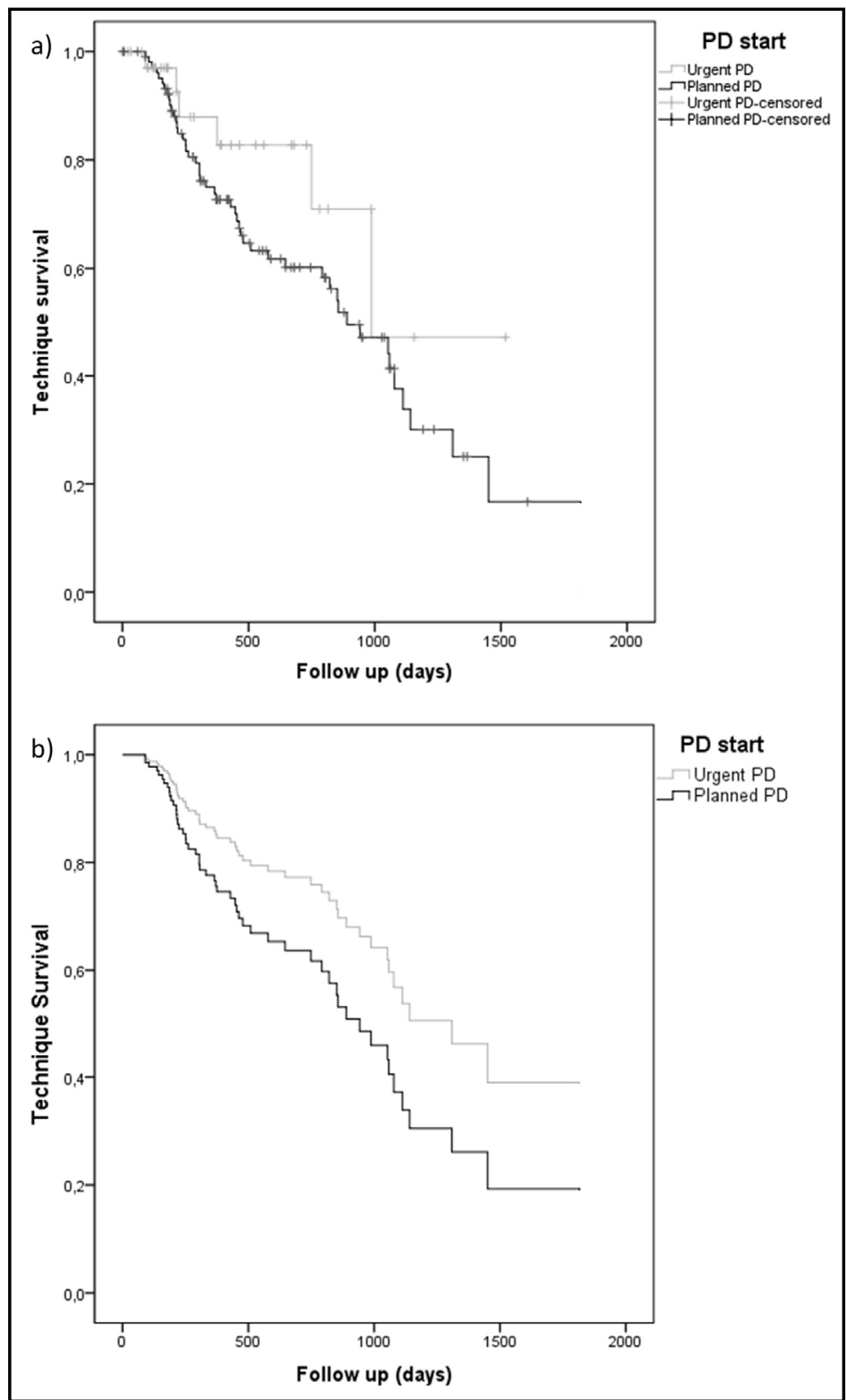

Fig. 1. Technique survival plot, according to PD start: a) non-adjusted Kaplan-Meier and b) Adjusted Cox regression analysis. 


\section{Kidney Blood Pressure Research}

\section{Discussion}

This study prospectively evaluated a cohort of patients that have either started early or planned PD. The main outcome was technique survival, which was not different between groups, whereas DM and BMI $>25 \mathrm{~kg} / \mathrm{m}^{2}$ were the only variables associated with PD dropout. The risks for peritonitis, catheter dysfunction or patient burnout were similar for early or planned-start PD.

Despite the efforts of avoiding unplanned dialysis initiation, approximately 60 to $70 \%$ of patients start dialysis under suboptimal conditions, without definitive vascular access or adequate follow-up by nephrologists [12]. Starting urgent HD with CVC is associated with poor outcomes, including higher risk of bloodstream infection, longer hospitalization and increased mortality in comparison to PD or planned-start HD [3, 4].

In the last decade, many studies have described the use of urgent-start PD, defined as initiation of dialysis within 14 days after catheter insertion [13]. Different protocols have been reported, including less than $72 \mathrm{~h}$ of break-in periods, $[6,8,10,11,14]$ and surgical catheter insertion $[5,7,15]$. In general, these studies have reported overall low complication rates $[3,11,15]$. Recently, Blake and Jain have proposed a new definition for urgent-start $\mathrm{PD}$, relegating this term exclusively to break-in periods shorter than $72 \mathrm{~h}$. Early start PD is a newly proposed term for patients who can wait from 3 to 14 days to initiate PD after catheter placement, since they do not truly require to start dialysis immediately [16].

Catheter dysfunction is a major concern in the context of early dialysis since delays in initiating dialysis procedure might potentially lead to severe complications. In urgent/early start PD, both surgical and percutaneous catheter insertions have been described and the prevalence of catheter dysfunction reported varied from 13.3 to $28.9 \%$ for surgical $[1,13$, 14 ] and from 11 to $25.7 \%$ for percutaneous placement [2, 5-8]. In this current study, $12.5 \%$ of early start group changed dialysis method for persistent catheter dysfunction, whereas early catheter replacement was more prevalent in planned-start PD. Such finding is more likely attributable to the characteristics of our service, since nephrologists usually insert more catheters in outpatients, which was more frequent in the planned-PD group.

Another concern regarding early PD break-in is peritonitis, an important cause of PD dropout and mortality [17]. Overall, studies have reported a prevalence of peritonitis ranging from 0 to $15.4 \%$ in urgent-start PD $[2,5,6,8,10]$. In this study, peritonitis led to conversion to HD in $7.5 \%$ of urgent-start group population, again not different from planned-start PD.

Peritoneal membrane failure was more frequent in planned-start HD. This is possibly a result of the longer follow-up period of this group. In early start PD, only $2.5 \%$ of patients had to quit PD for this reason, but median follow-up was only of 219 days. No patient withdrew from PD due to burnout in this group.

Undoubtedly, there are additional challenges when initiating PD urgently. The decision of choosing a dialysis method in a few days or even in a few hours, and the complexity of analyzing the best option under adverse conditions, could lead to a wrong choice, increasing cases of dropout from PD. Such technique survival has already been assessed elsewhere: Povlsen et al. analyzed technique survival at 3 months in their urgent-start PD patients compared to planned-start group and found similar results (86.7 vs. 90\%) [10]. In this present study, technique survival in early start PD group was $83.7 \%$, which is an encouraging result, given the longer follow-up period.

In order to adjust outcomes in both groups, once the follow-up period of planned-start PD group was longer in comparison to early-start group, we used the Cox regression analysis. In this case, only DM and BMI $>25 \mathrm{~kg} / \mathrm{m}^{2}$ were independently associated with technique

Table 4. Cox-regression analysis for PD failure. Covariates: BMI $>25$
$\mathrm{~kg} / \mathrm{m}^{2}$, diabetes mellitus and PD group (early start vs planned-start). BMI: body mass index, PD: peritoneal dialysis

\begin{tabular}{lccccc}
\hline \multirow{2}{*}{ Variables } & \multirow{2}{*}{ Beta coefficient } & \multirow{2}{*}{ t } & Hazard Ratio & \multicolumn{2}{c}{$95,0 \% \mathrm{CI}$} \\
& & & & Lower & Upper \\
\hline BMI $>25 \mathrm{~kg} / \mathrm{m}^{2}$ & -0.600 & 0.033 & 0.549 & 0.316 & 0.952 \\
Diabetes Mellitus & -0.723 & 0.013 & 0.485 & 0.274 & 0.859 \\
PD start & 0.580 & 0.184 & 1.785 & 0.759 & 4.198 \\
\hline
\end{tabular}

. 


\section{Kidney Blood Pressure Research}

failure, regardless of PD onset modality. However, even using an approach censuring patients to 1.5 years, which equals the period of follow-up between groups, does not modify the results of the Cox analysis (data not shown), reinforcing our findings of similar technique survival comparing early-start and planned-start PD. These findings are in line with data obtained from Australia and New Zealand Dialysis and Transplant Registry (ANZDATA): McDonald et al. found that BMI $>25 \mathrm{~kg} / \mathrm{m}^{2}$ was associated with technique failure [18]. More recently, See et al. described that the factors associated with technique failure, defined as transfer to HD or death, were age older than 70 years, DM or vascular disease, prior renal replacement therapy, late referral to a nephrology service or management in a smaller center [19].

Finally, two studies have assessed patient survival in urgent-PD: Lobbedez et al. found that patient survival at 1 year to be $83 \%$ [9], whereas Koch et al. described a survival of $69.7 \%$ after 6 months [14]. In our study, 10\% of early start PD group died during follow-up.

This study has some limitations. It was an observational, single-center study with a relatively small sample size. There was no randomization between groups and some outcomes could be influenced by differences regarding baseline characteristics, which it is impossible to overcome since early start cannot be avoided. Additionally, even though the follow-up period of planned-start was longer than the early start group, we have followed patients for a relatively longer period in comparison to other studies. Another strength of this study is the fact that all patients received similar medical treatment in both groups, which reinforces our findings. Finally, to the best of knowledge, this is the first study that included patients that fit the recently created definition of early PD start.

\section{Conclusion}

Despite the adverse scenario for initiating dialysis, early start PD had similar outcomes in comparison to planned-start PD, after a relatively long follow-up period. This study brings one more piece of evidence to support PD as an alternative treatment for renal replacement therapy in the context of urgent/early start dialysis.

\section{Acknowledgements}

We are grateful to all nursing staff who dedicated so much to the care of each patient.

The authors contributed to this article in the following way: Study design: BCS, HA, RME; data collect: EA; data analysis: BCS, RME; methodology: BCS, BJP, LC, CER, RJD; manuscript preparation: BCS, RME, RJD, HA.

\section{Disclosure Statement}

The authors have no conflicts of interest to declare.

\section{References}

1 Javaid MM, Khan BA, Subramanian S: Peritoneal dialysis as initial dialysis modality: A viable option for latepresenting end-stage renal disease. J Nephrol 2018; DOI:10.1007/s40620-018-0485-3.

72 Ghaffari A: Urgent-start peritoneal dialysis: A quality improvement report. Am J Kidney Dis 2012;59:400408.

3 Coentrao L, Santos-Araujo C, Dias C, Neto R, Pestana M: Effects of starting hemodialysis with an arteriovenous fistula or central venous catheter compared with peritoneal dialysis: A retrospective cohort study. BMC Nephrol 2012;13-88. 


\section{Kidney \\ Blood Pressure Research}

Silva et al.: Early Start PD Technique Survival

4 Perl J, Wald R, McFarlane P, Bargman JM, Vonesh E, Na Y, Jassal SV, Moist L: Hemodialysis vascular access modifies the association between dialysis modality and survival. J Am Soc Nephrol 2011;22:1113-1121.

5 Alkatheeri AM, Blake PG, Gray D, Jain AK: Success of urgent-start peritoneal dialysis in a large canadian renal program. Perit Dial Int 2016;36:171-176.

-6 Bitencourt Dias D, Mendes ML, Burgugi Banin V, Barretti P, Ponce D: Urgent-start peritoneal dialysis: The first year of brazilian experience. Blood Purif 2017;44:283-287.

-7 Javaid MM, Lee E, Khan BA, Subramanian S: Description of an urgent-start peritoneal dialysis program in singapore. Perit Dial Int 2017;37:500-502.

-8 Jo YI, Shin SK, Lee JH, Song JO, Park JH: Immediate initiation of capd following percutaneous catheter placement without break-in procedure. Perit Dial Int 2007;27:179-183.

-9 Lobbedez T, Lecouf A, Ficheux M, Henri P, Hurault de Ligny B, Ryckelynck JP: Is rapid initiation of peritoneal dialysis feasible in unplanned dialysis patients? A single-centre experience. Nephrol Dial Transplant 2008;23:3290-3294.

10 Povlsen JV, Ivarsen P: How to start the late referred esrd patient urgently on chronic apd. Nephrol Dial Transplant 2006;21:ii56-59.

11 Yang YF, Wang HJ, Yeh CC, Lin HH, Huang CC: Early initiation of continuous ambulatory peritoneal dialysis in patients undergoing surgical implantation of tenckhoff catheters. Perit Dial Int 2011;31:551-557.

12 Saran R, Li Y, Robinson B, Abbott KC, Agodoa LY, Ayanian J, Bragg-Gresham J, Balkrishnan R, Chen JL, Cope E, Eggers PW, Gillen D, Gipson D, Hailpern SM, Hall YN, He K, Herman W, Heung M, Hirth RA, Hutton D, Jacobsen SJ: US renal data system 2015 annual data report: Epidemiology of kidney disease in the united states. Am J Kidney Dis 2016;67:Svii, S1-305.

13 Arramreddy R, Zheng S, Saxena AB, Liebman SE, Wong L: Urgent-start peritoneal dialysis: A chance for a new beginning. Am J Kidney Dis 2014;63:390-395.

14 Koch M, Kohnle M, Trapp R, Haastert B, Rump LC, Aker S: Comparable outcome of acute unplanned peritoneal dialysis and haemodialysis. Nephrol Dial Transplant 2012;27:375-380.

15 Xu D, Liu T, Dong J: Urgent-start peritoneal dialysis complications: Prevalence and risk factorsAm J Kidney Dis 2017;70:102-110.

16 Blake PG, Jain AK: Urgent start peritoneal dialysis: Defining what it is and why it matters. Clin J Am Soc Nephrol 2018;13:1278-1279.

-17 Boudville N, Kemp A, Clayton P, Lim W, Badve SV, Hawley CM, McDonald SP, Wiggins KJ, Bannister KM, Brown FG, Johnson DW: Recent peritonitis associates with mortality among patients treated with peritoneal dialysis. J Am Soc Nephrol 2012;23:1398-1405.

18 McDonald SP, Collins JF, Johnson DW: Obesity is associated with worse peritoneal dialysis outcomes in the australia and new zealand patient populations. J Am Soc Nephrol 2003;14:2894-2901.

19 See EJ, Johnson DW, Hawley CM, Pascoe EM, Badve SV, Boudville N, Clayton PA, Sud K, Polkinghorne $\mathrm{KR}$, Borlace M, Cho Y: Risk predictors and causes of technique failure within the first year of peritoneal dialysis: An australia and new zealand dialysis and transplant registry (anzdata) study. Am J Kidney Dis 2018;72:188-197. 